\title{
Learning Curve in Robotics in ENT and Head and Neck Surgery
}

\author{
Kalpana Nagpal ${ }^{1 *}$, Malik ND ${ }^{1}$, Nishant Rana ${ }^{1}$, Harsh Madhok ${ }^{2}$ and Shalu Sharma ${ }^{1}$ \\ ${ }^{1}$ Department of ENT and Head and Neck Surgery, Apollo Hospitals, Indraprastha, India \\ ${ }^{2}$ Department of Anesthesiology, Apollo Hospitals, Indraprastha, India
}

\begin{abstract}
Surgical learning curve signifies how fast a surgeon acquires proficiency and improves the surgical skills. It provides an objective assessment of technical competence and depends on many factors. In ENT and Head and Neck robotic surgery, the learning curve is unique for a particular surgery. Multiple parameters of learning curve has been established and studied. An Otorhinolaryngologist performing few robotic surgeries will have longer learning curve as compared to someone who is performing these on a routine basis. Training the surgeons in robotics can achieve the expertise early as compared to conventional training. The data to support various parameters for evaluating learning curve in robotic surgery is scant in literature. There is a crucial role of training in making the learning curve shorter. A standardized training program can improve surgeon's performance, shorten a trainee's learning curve and improve patient outcomes.
\end{abstract}

Keywords: Learning curve; ENT and head and neck surgery; Robotic surgery; Robotic surgical training

\section{Introduction}

\section{The concept of learning curve}

Learning curve can be defined as the relationship between time and competence acquisition. It can be easily depicted with a simple curve having a starting point, a slope and a plateau [1]. Starting point is the initial phase of slow learning, when the surgeon starts performing new procedure,

\section{OPEN ACCESS}

${ }^{*}$ Correspondence:

Kalpana Nagpal, Department of ENT and Head and Neck surgery, Apollo Hospitals, Indraprastha, New Delhi,

India,

E-mail:kalps_apollo@yahoo.co.in

Received Date: 15 Jun 2020

Accepted Date: 02 Jul 2020

Published Date: 04 Jul 2020

Citation:

Nagpal K, Malik ND, Rana N, Madhok

$H$, Sharma S. Learning Curve in Robotics in ENT and Head and Neck Surgery. Am J Otolaryngol Head Neck Surg. 2020; 3(5): 1102.

Copyright $\odot 2020$ Kalpana Nagpal. This is an open access article distributed under the Creative Commons Attribution License, which permits unrestricted use, distribution,

and reproduction in any medium, provided the original work is properly

cited. finds it more difficult and takes longer time. Then follows a slope of quick learning where rapid improvement is seen and depends on how a surgeon learns the procedure. Finally, when the surgeon deems enough experience, a plateau stage is reached.

Surgical learning curve signifies how fast a surgeon acquires proficiency and improves the surgical skills with chronological number of cases he/she performs. Learning curve also provides an objective assessment of technical competence in performing a particular surgery or procedure.

In surgical field learning curve depends on many factors like training intensity, surgeon's team, surgical procedure, operative time, technical efficiency, patient outcome and previous experiences of the surgeon [2-4]. The learning curve is used for various purposes besides as a technique of cost reduction like work scheduling and time management, material requirement planning, training program for young surgeons, cost estimation and capital requirement planning. In this paper, our objective is to review the impact of learning curve of robotic surgeries for various procedures in ENT and head and neck surgery and also our tertiary care institute's experience.

\section{Learning curve in ENT and head and neck robotic surgery}

The learning curve of robotic surgery in Otorhinolaryngology has not yet been clearly defined and not many studies have stressed on it till now. In India not even a handful of ENT surgeons are doing it.

In ENT and Head and Neck robotic surgery, the learning curve is unique for a particular surgery because some robotic procedures are intrinsically more complicated and demanding than others. For example, achieving success in Transoral Robotic Surgery (TORS) can take more time and more number of cases as compared to trans-axillary robotic thyroidectomy and hence the learning curve of the latter is considered to be less steep [5]. Achieving expertise in robotic neck dissection may require more cases than required for robotic thyroidectomy. Similarly learning curve for robotic thyroidectomy can be unique for different approaches and thus any new surgical technique is 
contingent to a learning curve. So it can be used to compare different surgical approaches like time required to attain expertise in each approach. For example, transaxillary thyroidectomy can have shorter learning curve as compared to retro auricular, facelift or transoral approach as they are technically more demanding.

A freshly trained Otorhinolaryngologist performing robotic surgeries in ENT and head and neck surgery under the guidance of well-trained senior robotic surgeon will have different output as compared to a senior Otorhinolaryngologist who started performing robotic surgeries without any expert guidance. Although not much studies are available in ENT and Head and Neck Surgery per se, but according to a study in an urology institute (Vattikuti Urology Institute, USA), a fellow who is observing robotic surgeries with minimal experience in conventional surgeries may have a shorter learning curve for robotic surgeries as compared to someone with more experience in conventional surgeries [6].

\section{Role of training in learning curve}

The intensity of the training has a major impact on the output and likely shortens the learning curve. The importance of the specialized training programs in robotics should be emphasized. Robotic technology has unique learning curve profile for training surgeons and the criteria for credentialing should be well defined. Various studies have proven the enhancement of robotic surgical skills after systematic training with surgical robotic system [7-9]. The robotic training should be in a systematic and stepwise curriculum starting from observation and assisting followed by robotic console training and independent practice under supervision of expert robotic surgeons, so that the learning curve can be effectively minimized. Each step to be mastered independently before proceeding with the final surgery. This systematic training will minimize the learningassociated morbidity, helps in developing more skillful movements of surgeon, improvement in machine and tooling and better operation sequences.

Simulator-based training can make an effective impact on developing and improving the robotic skills in ENT and Head and Neck Surgery. Simulation based training can improve the initial surgical learning phase of training, early stabilization in performance and thereby minimizing performance errors. It is not only beneficial to the trainee, but an experienced robotic surgeon can also improve their skills in the operating room by practicing simulator-based difficult exercises. A stepwise learning, initiating from mentoring and subsequently to proctorship will be influential. Still there is a lack of standardization in training of robotic surgeons but every possible effort should be made to shorten the learning curve.

In India there is a lack of formal head and neck robotic training centers in India and lack of dual consoles and cadaver labs is one of the reasons for longer learning curve among young surgeons. That is why India is not able to produce a lot of robotic surgeons in this field.

\section{Probable parameters of learning curve}

Few published studies have considered console or operating time as a measure of surgeon's improvement and learning curve for a particular procedure $[10,11]$. We can assume that the operating time becomes less as the surgeon performs any particular surgery on a routine basis more frequently and also it takes around 20 to 40 cases to declare the end of the learning curve. Likewise in ENT and head and neck surgery, operating or console time is short for TORS for OSA (obstructive sleep apnea) whereas for neck dissection, it is relatively longer. The parameters like conversion rates, intraoperative and postoperative complication rates including functional outcomes, surgeon's ergonomics, hospital length of stay and training curve plateau can be used for comparing the learning curve.

Cumulative Sum (CUSUM) learning curves takes failure rate (such as positive margin in malignant cases) in account for monitoring quality of surgery and accepted as a relevant measure of learning curve [11]. Albergotti et al. used CUSUM in their study and concluded that 15 to 40 cases of TORS for squamous cell carcinoma of the oropharynx are required to reach the peak of learning curve and is always surgeon specific.

No doubt, an Otorhinolaryngologist performing few robotic surgeries will have longer learning curve as compared to someone who is performing these on a routine basis. The outcome of the surgeries can be evaluated as the experience of the robotic surgeon increases. The complication rates and operative times can be compared from earlier surgeries performed by the same surgeon with that performed later in the learning curve.

With the introduction of any new surgical technique or approach, there is an anticipated learning where robotic surgeon achieves expertise in the technique which has not been developed completely yet. It is typically steepest in the beginning, when the surgeon is gaining new skills and eventually achieves a plateau stage with increase in frequency of cases when operating time, complication rates and outcomes have stabilized. For these procedures and approaches the learning curve will be different from well-established surgical technique. Here surgical process can be taken as a parameter to measure the learning curve.

\section{Transoral robotic surgery (TORS)}

With the introduction of Transoral Robotic Surgery (TORS) in ENT and head and neck surgery, the technique is being increasingly used for treating a range of benign and malignant lesions of pharynx and larynx transorally [12].

In 2015, the American Head and Neck Society (AHNS) Education Committee, American Academy of OtolaryngologyHead and Neck (AAO-HNS) surgery Robotic Task Force, and AAO-HNS Sleep Disorders Committee proposed the guidelines for training and credentialing robotic head and neck surgeons, which suggested a minimum 20 cases ( 10 bedside and 10 console) of TORS for residents and fellows in TORS training program or 2 proctored cases for surgeons who completed approved training courses [13]. O' Malley and Weinstein suggested that for TORS, one should perform minimum 20 cases to reach the plateau of the learning curve [14]. A 4-year (2013-2017) learning experience of Isenberg et al. in TORS for Oropharyngeal Squamous Cell Carcinoma (OPSCC) showed a decline in overall complication rates due to surgical learning experience with time [15].

Because of high costs of surgical Robot and the instruments like FK-WO Laryngo-Pharyngoscope retractor there are not many robotic head and neck centers in India. Only few large corporate hospitals have the facility. As we are performing more number of cases with time in our tertiary care centre, we are experiencing improvement in the technique and approaching the plateau of the learning curve. We are improving in terms of operating time, patient outcomes, decrease of hospital stay, efficiency in the resection of the tumor or lesion and decrease in overall complication rates. 


\section{Transoral robotic surgery (TORS) for obstructive sleep apnea (OSA)}

It has been observed in our personal experience, that TORS for OSA seems to have shorter learning curve among all ENT and head and neck robotic surgeries and plateau can be achieved more rapidly as compared to other conventional open approaches available for OSA.

One of the limitations for open approaches in OSA is its steep learning curve. Open approaches are limited by instrument maneuverability, high morbidity and longer hospital stay. Use of da Vinci system alleviate these disadvantages. The current robotic system provides an excellent stereoscopic vision which has a distinct impact on learning of TORS. Other advantages being depth perception, excellent instrumental maneuverability, elimination of physiological tremors and better ergonomics [16]. Patient outcomes in terms of blood loss, hospital stay and improvement in Apnea-Hypopnea Index (AHI) after TORS are few variables to measure the learning and can be compared against open approaches. During our 3-year learning experience (November 2016 to November 2019) of TORS for OSA with the da Vinci system, we have observed a change and improvement in terms of complications, hospital stay and operative times. More than 100 TORS has been performed in our tertiary institute and a constant improvement has been noticed in last 70 to 80 cases. We were able to perform wider resections of base of tongue without complications with more confidence and this resulted in excellent outcomes.

\section{Robotic thyroidectomy}

Thyroidectomy is one of the common procedures performed by an Otorhinolaryngologist. It is performed more commonly for benign thyroid nodules or colloid goiter. The conventional cervical approach for thyroidectomy leaves a visible inevitable bad scar over neck. And now patients are much aware that scar-less thyroidectomy is being performed in robotic centers and opting for the same for better cosmesis but in India it numbers less than TORS because of lack of awareness in public and criticism in physician community.

Robotic thyroidectomy via any approach remains technically demanding and more challenging than conventional thyroidectomy. But provide several added advantages over conventional and endoscopic techniques like stereoscopic 3D magnified vision, motion scaling, excellent ergonomics and meticulous surgery can be performed using endowrist arms having 7 degrees of freedom [17]. Various studies have shown advantages of robotic thyroid surgery over conventional, because of its excellent cosmetic outcome, reduced neck pain and a minimal swallowing discomfort $[18,19]$.

Robotic thyroidectomy is performed via various approaches. Transaxillary approach was the first approach developed for robotic thyroidectomy; followed by retroauricular and facelift approaches. For the last few years transoral approach is being developed for accessing thyroid gland. Creating a sufficient 'working space' in robotic thyroidectomy is one aspect which can modify the learning curve. The surgeon must learn the extent of working space required for performing the surgery successfully without collisions of interarms of robot.

When operative time is considered as a parameter for thyroidectomy, robotic thyroidectomy is limited by a steep learning curve. Generally operative time is the time from the incision to the closure. But for robotic thyroidectomy, operative time is calculated from making the tunnel, resecting the gland till closure of the tunnel. Various studies have shown more operative time for robotic thyroidectomy as compared to conventional thyroidectomy [20].

Kim et al. [21] in their study performed 100 cases of robotic total thyroidectomy via bilateral axillo-breast approach; they concluded that robotic total thyroidectomy was performed effectively and safely after 40 cases. Kang et al. [22] showed that 45 to 50 robotic thyroidectomy procedures are required to reach the plateau of $60 \mathrm{~min}$ of console time. In another study Lee et al. concluded that, performing 50 robotic total thyroidectomy by a single surgeon markedly decreases the operation time and complication rate. Park et al. [23] showed in their institutional study that recently graduated fellow surgeons with little or no experience in endoscopic surgery performed better and the results were comparable to those of experienced surgeons after performing 20 cases of robotic thyroidectomy. According to Giannopoulos et al. [20], transaxillary robotic thyroidectomy has a steep learning curve and progress can be achieved with the first 10 cases in terms of operating times.

\section{Robot-assisted neck dissection}

Robot-Assisted Neck Dissection (RAND) is the newly developed robotic technique for regional metastasis in head and neck cancers however very limited studies are available in this context [24-27]. Considering the technical complexity of the procedure, even a seasoned head and neck surgeon might have to experience a long learning curve, especially if they do not have enough endoscopic surgery experience.

In a 3 year (2010 to 2013) single institutional study, Kim et al. [28] observed a significant decrease in mean operation time with experience, mean length of hospital stay and mean amount of drainage. They analyzed both Modified Radical Neck Dissection (MRND) and the Selective Neck Dissection (SND) in their study.

In our tertiary care hospital we have not been doing robotic neck dissection but planning to start in a couple of months.

\section{Conclusion}

The question arises whether a fresh ENT surgeon in his/her early phase of learning curve can attain the plateau at the same pace as a well experienced surgeon. Many skilled ENT robotic surgeons believe that training the surgeons in robotics can achieve the expertise early as compared to conventional training. There is no standard method of measurement of learning curve and it is totally dependent upon the surgeon, when he becomes dexterous with the robotic procedure. The data to support various parameters for evaluating learning curve in robotic surgery is scant in literature. Only few studies of learning curve assessment are available in ENT and head and neck robotics. There is a need to set established parameters and quantitative methods for standardizing the assessment of learning curve in ENT and head and neck robotic surgery. The parameters of learning curve assessment in context to disease extent and pathology (benign or malignant) should be established separately.

However, as per our institutional experience there seems to be a general agreement that an otolaryngologist can safely perform successful robotic procedure after 25 to 30 cases but this can vary from procedure to procedure. Simulation-based exercises and a standardized training program can improve surgeon's performance, shorten a trainee's learning curve and improve patient outcomes. 


\section{Acknowledgement}

We would like to thank department of ENT and Head and Neck surgery, Indraprastha Apollo Hospitals, New Delhi India.

\section{References}

1. Andolfi C, Umanskiy K. Mastering robotic surgery: Where does the learning curve lead us? J Laparoendosc Adv Surg Tech A. 2017;27(5):4704 .

2. Mazzon G, Sridhar A, Busuttil G, Thompson J, Nathan S, Briggs T, et al. Learning curves for robotic surgery: A review of the recent literature. Curr Urol Rep. 2017;18(11):89.

3. Cook JA, Ramsay CR, Fayers P. Using the literature to quantify the learning curve: A case study. Int J Technol Assess Health Care. 2007;23(2):255-60.

4. Subramonian K, Muir G. The 'learning curve's in surgery: What is it, how do we measure it and can we influence it? BJU Int. 2004;93(9):1173-4.

5. Lee J, Yun JH, Nam KH, Soh EY, Chung WY. The learning curve for robotic thyroidectomy: A multicenter study. Ann Surg Oncol. 2011;18(1):226-32.

6. Kaul S, Shah NL, Menon M. Learning curve using robotic surgery. Curr Urol Rep. 2006;7(2):125-9.

7. Gulbins H, Boehm DH, Reichenspurner H, Arnold M, Ellgass R, Reichart B. 3D-visualization improves the dry-lab coronary anastomoses using the Zeus robotic system. Heart Surg Forum. 1999;2(4):318-24.

8. Mohr FW, Falk V, Diegeler A, Walther T, Gummert JF, Bucerius J, et al. Computer-enhanced "robotic" cardiac surgery: Experience in 148 patients. J Thorac Cardiovasc Surg. 2001;121(5):842-53.

9. Rassweiler J, Binder J, Frede T. Robotic and telesurgery: Will they change our future? Curr Opin Urol. 2001;11(3):309-20.

10. Khan N, Abboudi H, Khan MS, Dasgupta P, Ahmed K. Measuring the surgical 'learning curve': Methods, variables and competency. BJU Int. 2014;113(3):504-8

11. Mazzon G, Sridhar A, Busuttil G, Thompson J, Nathan S, Briggs T, et al. Learning curves for robotic surgery: A review of the recent literature. Curr Urol Rep. 2017;18(11):89.

12. White HN, Frederick J, Zimmerman T, Carroll WR, Magnuson JS Learning curve for transoral robotic surgery: A 4-year analysis. JAMA Otolaryngol Head Neck Surg. 2013;139(6):564-7.

13. Gross ND, Holsinger FC, Magnuson JS, Duvvuri U, Genden EM, Ghanem TA, et al. Robotics in otolaryngology and head and neck surgery: Recommendations for training and credentialing: A report of the 2015 AHNS education committee, AAO-HNS robotic task force and AAOHNS sleep disorders committee. Head Neck. 2016;38(Suppl 1):E151-8.

14. Weinstein GS, O'Malley BW. Trans Oral Robotic Surgery (TORS). Plural Publishing; 2011.

15. Hutcheson KA, Warneke CL, Yao CM, Zaveri J, Elgohari BE, Goepfert $\mathrm{R}$, et al. Dysphagia after primary transoral robotic surgery with neck dissection $v s$. nonsurgical therapy in patients with low-to intermediaterisk oropharyngeal cancer. JAMA Otolaryngol Head Neck Surg. 2019;145(11):1053-63.
16. Hay A, Migliacci J, Zanoni DK, Boyle JO, Singh B, Wong RJ, et al. Complications following transoral robotic surgery (TORS): A detailed institutional review of complications. Oral Oncol. 2017;67:160-6.

17. Choi JY, Youn YK. Vision of thyroid surgery: Past, present and future. J Korean Thyroid Assoc. 2012;5(1):1-5.

18. Lee J, Nah KY, Kim RM, Ahn YH, Soh EY, Chung WY. Differences in postoperative outcomes, function, and cosmesis: Open versus robotic thyroidectomy. Surg Endos. 2010;24(12):3186-94.

19. Tae K, Ji YB, Jeong JH, Lee SH, Jeong MA, Park CW. Robotic thyroidectomy by a gasless unilateral axillo-breast or axillary approach: Our early experiences. Surg Endos. 2011;25(1):221-8.

20. Giannopoulos G, Kang SW, Jeong JJ, Nam KH, Chung WY. Robotic thyroidectomy for benign thyroid diseases: A stepwise strategy to the adoption of robotic thyroidectomy (gasless, transaxillary approach). Surg Laparosc Endosc Percutan Tech. 2013;23(3):312-5.

21. Kim WW, Jung JH, Park HY. The learning curve for robotic thyroidectomy using a bilateral axillo-breast approach from the 100 cases. Surg Laparosc Endosc Percutan Tech. 2015;25(5):412-6.

22. Kang SW, Lee SC, Lee SH, Lee KY, Jeong JJ, Lee YS, et al. Robotic thyroid surgery using a gasless, transaxillary approach and the da Vinci S system: The operative outcomes of 338 consecutive patients. Surgery. 2009;146(6):1048-55.

23. Park JH, Lee J, Hakim NA, Kim HY, Kang SW, Jeong JJ, et al. Robotic thyroidectomy learning curve for beginning surgeons with little or no experience of endoscopic surgery. Head Neck. 2015;37(12):1705-11.

24. Kim WS, Lee HS, Kang SM, Hong HJ, Koh YW, Lee HY, et al. Feasibility of robot-assisted neck dissections via a transaxillary and retroauricular ("TARA") approach in head and neck cancer: Preliminary results. Ann Surg Oncol. 2012;19(3):1009-17.

25. Lee HS, Kim WS, Hong HJ, Ban MJ, Lee D, Koh YW, et al. Robot-assisted Supraomohyoid neck dissection via a modified face-lift or retroauricular approach in early-stage cN0 squamous cell carcinoma of the oral cavity: A comparative study with conventional technique. Ann Surg Oncol. 2012;19(12):3871-8.

26. Kim CH, Koh YW, Kim D, Chang JW, Choi EC, Shin YS. Robotic-assisted neck dissection in submandibular gland cancer: Preliminary report. J Oral Maxillofac Surg. 2013;71(8):1450-7.

27. Shin YS, Hong HJ, Koh YW, Chung WY, Lee HY, Hong JM, et al. Gasless transaxillary robot-assisted neck dissection: A preclinical feasibility study in four cadavers. Yonsei Med J. 2012;53(1):193-7.

28. Kim WS, Ban MJ, Chang JW, Byeon HK, Kim H, Han JH, et al. Learning curve for robot-assisted neck dissection in head and neck cancer: A 3-year prospective case study and analysis. JAMA Otolaryngol Head Neck Surg. 2014;140(12):1191-7. 